\title{
Recurrent pituitary apoplexy due to two successive neoplasms presenting with ocular paresis and epistaxis
}

\author{
Stephanie Teasdale', Fahid Hashem ${ }^{1}$, Sarah Olson ${ }^{2}$, Benjamin Ong ${ }^{3}$ \\ and Warrick J Inder ${ }^{1,4}$
}

Departments of ${ }^{1}$ Diabetes and Endocrinology ${ }^{2}$ Neurosurgery ${ }^{3}$ Radiology, Princess Alexandra Hospital, Brisbane, Queensland, Australia

${ }^{4}$ School of Medicine, The University of Queensland, Brisbane, Queensland, Australia

Correspondence

should be addressed

to W J Inder

Email

warrick.inder@

health.qld.gov.au

\section{Summary}

A case of recurrent pituitary apoplexy is described in a 72-year-old man who initially presented with haemorrhage in a nonfunctioning pituitary adenoma. Five years later, he re-presented with a severe pituitary haemorrhage in an enlarging sellar mass invading both cavernous sinuses causing epistaxis and bilateral ocular paresis. Subsequent histology was consistent with a sellar malignant spindle and round cell neoplasm. Multiple pituitary tumours have previously been reported to coexist in the same individual, but to our knowledge this is the only case where two pathologically distinct pituitary neoplasms have sequentially arisen in a single patient. This case is also notable with respect to the progressive ocular paresis, including bilateral abducens nerve palsies, and the presentation with epistaxis.

\section{Learning points:}

- Ocular paresis in pituitary apoplexy can result from tumour infiltration of nerves, or by indirect compression via increased intrasellar pressure.

- Epistaxis is a very rare presentation of a pituitary lesion.

- Epistaxis more commonly occurs following trans-sphenoidal surgery, and can be delayed.

\section{Background}

Pituitary apoplexy is a relatively rare complication of pituitary tumours (1). Most published series comprise 30-60 patients, predominantly in pituitary adenomas, with isolated case reports outlining less common presentations in other sellar lesions. In recent years, conservative management has been proposed initially, with the limited evidence available suggesting that surgery can be reserved for cases where there is persisting visual acuity or visual field defects, or altered level of consciousness. The outcome of ocular paresis and pituitary function do not appear to be altered by surgical intervention (1).
There have been reports of recurrent pituitary apoplexy occurring in the same patient, but in general it is thought that tumour recurrence after pituitary apoplexy is less common than after elective surgery for an uncomplicated pituitary adenoma (2). The case presented here is notable for the following points:

i) The pattern of ocular paresis and in particular bilateral abducens nerve palsy.

ii) Presentation of pituitary haemorrhage with epistaxis.

iii) The sequential occurrence of two pathologically distinct pituitary neoplasms in the same individual. 
iv) The ultimate diagnosis of a sellar malignant spindle and round cell neoplasm.

\section{Case presentation}

A 72-year-old man who ran a beef cattle farm presented in 2008 with severe-sudden onset headache, nausea and vomiting. His blood pressure was $118 / 55 \mathrm{mmHg}$, with heart rate 59 b.p.m., oxygen saturations $97 \%$ on room air. He had hazy vision but no visual field deficit at initial presentation. The computed tomography (CT) scan of head demonstrated a $2.5 \mathrm{~cm}$ sellar and suprasellar mass compressing the optic chiasm. The mass was hyperdense and the pituitary fossa was expanded, indicating haemorrhage into a pituitary tumour. The pituitary lesion measured $17 \times 14 \mathrm{~mm}$, extending to the surface of the optic chiasm with haemorrhage. He had a CT angiogram which ruled out aneurysm. His vision worsened a day after his initial presentation, with development of a bitemporal haemianopia, worse on the left than the right. There were no symptoms or signs of anterior pituitary hormone insufficiency or excess.

He underwent urgent trans-sphenoidal surgery. Preoperative evaluation of pituitary function were difficult to interpret given they were drawn at $2130 \mathrm{~h}$ and in light of probable exogenous steroids given earlier. However, there was evidence of hypogonadotrophic hypogonadism and hypoprolactinaemia. The biochemical parameters included cortisol $496 \mathrm{nmol} / 1$, adrenocorticotropin (ACTH) $<10 \mathrm{ng} / \mathrm{l}$, testosterone $0.5 \mathrm{nmol} / \mathrm{l}$ (9-35), folliclestimulating hormone (FSH) $3.1 \mathrm{IU} / 1$, luteinising hormone $0.9 \mathrm{IU} / 1$ (1-9), thyroid-stimulating hormone $2.4 \mathrm{mIU} / \mathrm{l}$, free thyroxine $\left(\mathrm{T}_{4}\right) 9.9 \mathrm{pmol} / \mathrm{l}$, free tri-iodothyronine $3.8 \mathrm{pmol} / 1$, growth hormone $(\mathrm{GH}) 2.9 \mathrm{mIU} / 1$, insulin-like growth factor $110 \mathrm{nmol} / 1$, prolactin $36 \mathrm{mIU} / 1$ (56-278).

Histological examination demonstrated haemorrhagic infarction of the pituitary (Figure 1). Reticulin stain was difficult to interpret to ascertain the presence of a neoplasm, but ultimately it was concluded that there was a partially infarcted pituitary adenoma, with positive immunohistochemistry staining for FSH (weak), thyrotrophin and luteinizing hormone. There was no staining with ACTH, GH, prolactin and cam 5.2.

Visual fields postoperatively improved to a left superior quadrantanopia to confrontation, with normal extraocular movements. Formal visual fields 4 months postoperatively showed improved but residual upper temporal quadrant loss and right inferior quadrant loss. This continued to improve with minimal deficit at 1 year post operation. He developed postoperative panhypopituitarism, requiring replacement therapy with $\mathrm{T}_{4} 100 \mu \mathrm{g}$ daily, cortisone acetate $12.5 / 5 \mathrm{mg}$ daily, testosterone gel $50 \mathrm{mg}$ daily via the transdermal route.

The patient had no remarkable medical history before the initial presentation, but in the succeeding years was diagnosed with type 2 diabetes mellitus, hypertension, obesity (BMI $31 \mathrm{~kg} / \mathrm{m}^{2}$ ), hypercholesterolaemia, bilateral cataracts, for which he underwent surgery on the right eye in 2012, transient ischaemic attack requiring unilateral carotid angioplasty and a single-tonic clonic seizure. Medications included metformin, atorvastatin, sodium valproate and an antihypertensive.

Magnetic resonance imaging (MRI) scanning 3 months postoperatively demonstrated removal of the tumour with minor residual soft tissue in the sellar floor, which remained stable on and follow up imaging for 2 successive years, with a plan for another MRI after a further 3 year interval. He continued to report reasonable energy levels, and felt well on hormone replacement therapy.

In 2013, nearly 5 years after his initial presentation he presented with a 3-day history of a mild occipital headache with vomiting and visual disturbance. Examination demonstrated a partial right oculomotor nerve palsy, with partial ptosis, diplopia but pupil sparing. Visual fields were intact to confrontation. Other cranial nerves were normal. MRI demonstrated T1 hyperintensity and abnormal soft tissue in the sella and right cavernous sinus, suggesting haemorrhage, possibly into underlying tumour. The differential included an inflammatory or infective lesion. Follow-up imaging demonstrated resolution of the T1 hyperintensity, consistent with evolution of blood products. There was also noted to be a depressed optic chiasm, which was not different to previous imaging. The cranial nerve signs resolved on dexamethasone.

Three weeks later, the man re-presented with a 2-day history of severe headache, vomiting, tunnel vision and diplopia. The right partial oculomotor nerve palsy had returned, but there also developed bilateral abducens nerve palsies. MRI demonstrated a mild increase in the sellar soft tissue mass, and notably increased tumour invading the right cavernous sinus compared with the scan 3 weeks earlier. At the time, the most likely diagnosis was thought to be further evolution of previous haemorrhage in underlying recurrent pituitary adenoma, but because of the atypical presentation, the differential remained wide.

The case was discussed on three occasions in a multidiscliplinary meeting involving endocrinologists, neurosurgeons, neuroradiologists and neuropathologists. 
The endocrine team suggested biopsy of the lesion, but it was not undertaken as the patient settled with conservative management.

Nearly 2 months after the first presentation in 2013, he presented again, this time with 3 weeks of worsening epistaxis and with a significant drop in haemoglobin level from $149 \mathrm{~g} / \mathrm{l}$ a month earlier to $91 \mathrm{~g} / \mathrm{l}$. He also had a 1 week history of diplopia, with worsening headaches, vomiting and falls. Cortisone acetate was increased by the patient with no improvement in his symptoms. Examination demonstrated bilateral ptosis, bilateral ophthalmoplegia on all movements, complete on the right, dyscoria and only finger counting for acuity. The visual fields were not documented. Thus, there was a bilateral optic, oculomotor and trochlear nerve palsy, as a right and likely also left abducens nerve palsies. CT head, MRI head and CT-angiogram were performed. The imaging demonstrated an atypical, rapidly growing lesion with now bilateral cavernous sinus involvement, with extension posteriorly into the pre-pontine cistern. There was subtle reduction in calibre of the cavernous internal carotid arteries, suggesting extrinsic compression by the sellar mass. The patient underwent debulking left sphenoidotomy $1800 \mathrm{~h}$ later. Operative notes documented a tumour arising from the pituitary fossa eroding through the floor of the sella, filling the right sphenoid sinus, destroying the clivus or posterior wall of the sphenoid sinus and encasement of bilateral parasellar carotids.

\section{Investigation}

Histology on the 2013 tumour (Figure 2):

i) Closely packed spindle or rounded cells with high N/C ratio, associated with thin-walled branching vessels and no particular architectural arrangement.

ii) Focal staining for $\mathrm{CD} 34$ and epithelial membrane antigen (EMA). Cytokeratins negative; S100, desmin and BCL2 negative; SYT translocation negative; smooth muscle actin (SMA) positive. Mitotic rate ten/10 high power field. Ki67 proliferative index 5-10\%.

iii) Initially labelled as a haemangiopericytoma of sinonasal type or glomangiopericytoma.

iv) A further specialist opinion did not favour haemangiopericytoma as immunostatins for CD34 and STAT6 were negative. Synovial sarcoma was considered as there was striking positivity for TLE1, but ruled out when stains for EMA and AE1/AE were negative, and FISH testing was also noted to be negative. v) Additional stains for SMA, S-100 protein, GFAP and SOX10 were all negative.

vi) A final diagnosis was made of malignant spindle and round cell neoplasm, without clear evidence of any specific line of differentiation. An unusual feature was remarked upon large stromal blood vessels adjacent to the tumour, showing prominent proliferation of plump ovoid pale-staining pericytic cells (SMA-positive). This feature is of uncertain significance.

\section{Treatment}

Following debulking sphenoidotomy, the patient underwent radiotherapy with 54 Gray over 30 fractions, aiming for local control and haemostasis.

\section{Outcome and follow-up}

Review 1 month after radiotherapy demonstrated some recovery of cranial nerve function, but with residual partial right oculomotor, complete right abducens and mild left abducens palsies. MRI was repeated immediately after radiotherapy, but was essentially unchanged in light of its temporal proximity to surgery (Figs. 3 and 4).

He reported progressive right scapula pain, and CT chest demonstrated seven rounded soft tissue masses in the lungs, the largest measuring 13-mm diameter, most likely metastases, but no apparent lymph node involvement, no effusions and no evidence of bony metastases. He died 2 months after this review.

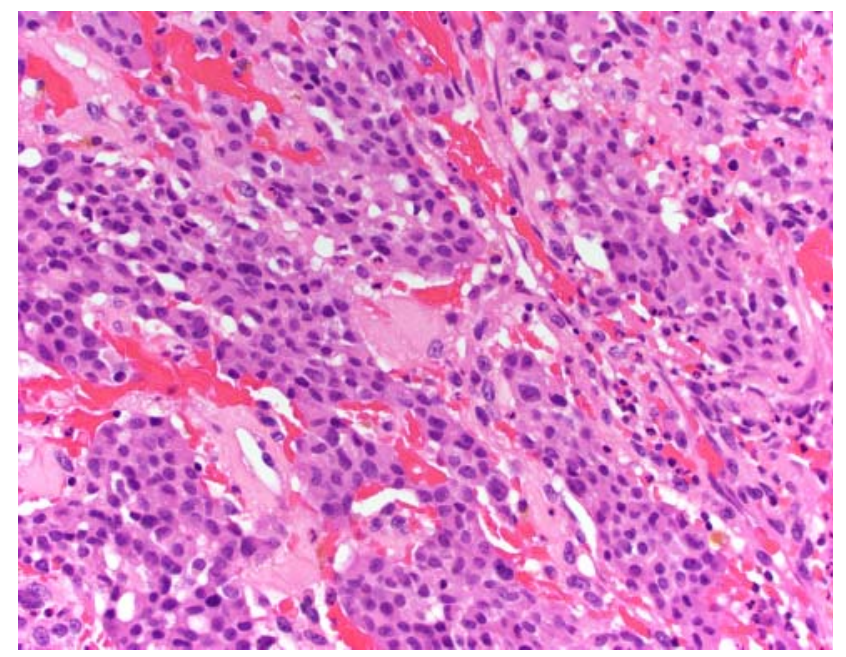

Figure 1

Histology 2008. High-power view of viable pituitary adenoma showing epithelioid cells with a nested growth pattern. 


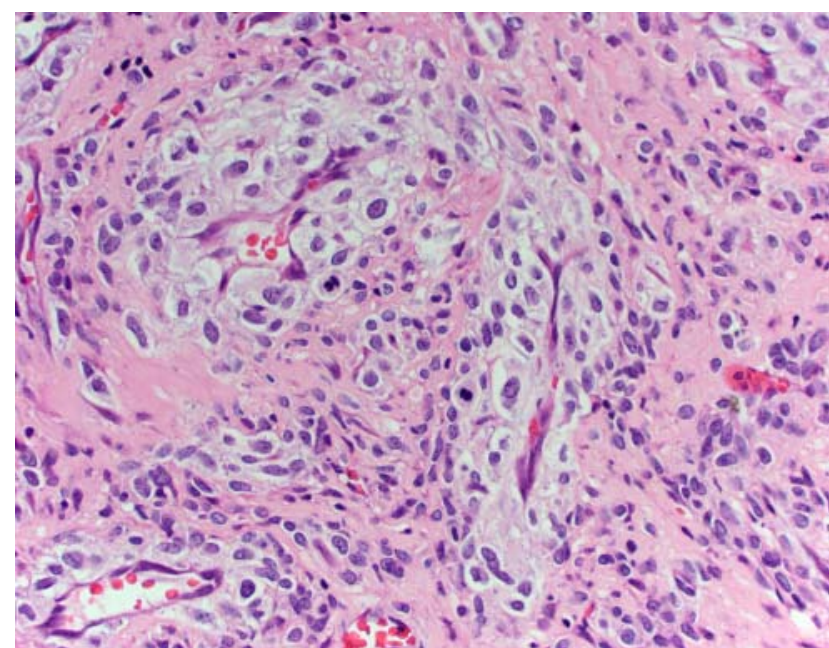

Figure 2

Histology 2013. High-power view of the malignant spindle and round-cell tumour with several mitoses in the centre.

\section{Discussion}

\section{Cranial nerve palsies}

Older series before availability of MRI reported cranial nerve palsies in $14-33 \%$ of pituitary tumours, many associated with tumour invasion into the cavernous sinus (3). Cranial nerve palsies can also occur in the absence of tumour invasion. When cavernous sinus invasion is excluded, the rate of cranial nerve palsies is reported to be $2.5 \%$, and present with pituitary apoplexy (4). The mechanism by which cranial nerve palsies arise in the absence of cavernous sinus invasion is due to mechanical compression from the transmitted pressure related to sellar swelling in the days following pituitary haemorrhage. The oculomotor nerve is the most frequently affected nerve, followed by the trochlear and then the abducens nerve. The reasons for this are reportedly due to the relatively thin meningeal envelope surrounding the oculomotor nerve, and its location abutting the firm dural wall of the lateral cavernous sinus. In contrast, the abducens nerve traverses the cavernous sinus adjacent to the internal carotid artery, and in this location seems not as amenable to compression. It is thought that abducens nerve palsy occurs on expansion of the tumour posteriorly to involve the petrous temporal bone. At the petrous temporal bone, the abducens nerve is tethered to a firm structure, and is thus more amenable to disruption.

Bilateral cranial nerve palsies are very rare. A search of the literature identified three cases of bilateral oculomotor nerve palsy, one which followed coronary artery bypass graft surgery and another which spontaneously resolved (5). There were eight cases of total ophthalmoplegia. (3) (6) (7) (8) (9).

In our case, the presentation with unilateral oculomotor nerve palsy and bilateral abducens nerve palsies 3 weeks after the first cranial nerve palsy was associated with unilateral cavernous sinus invasion and haemorrhage. There was no evidence yet on imaging that there had been tumour extension posteriorly, although this may have been just a limitation of MRI capability. A further month later when the ocular paresis had worsened, MRI evidenced posterior tumour invasion, which could readily explain the cranial nerve signs.

\section{Epistaxis}

Post-operative epistaxis complicates around 3\% of transsphenoidal surgery. The majority present within 2 years of surgery and generally resolve promptly with nasal packing unless on antiplatelet agents (10). More rarely pseudoaneurysms can form as a complication of intraoperative vascular injury, with subsequent delayed epistaxis.

Our literature search reveals only 15 previous reported cases of pituitary tumours that present with epistaxis (11). These presentations resulted variably from bleeding from a pituitary aneurysm, tumour invasion of the carotids or tumour invading the nasal cavity.

An arterial bleed in our patient is thought unlikely. MRI did show carotid artery involvement, but he did not require embolisation. Bleeding ceased after removal of the fragile tumour which showed necrosis and intra-tumour
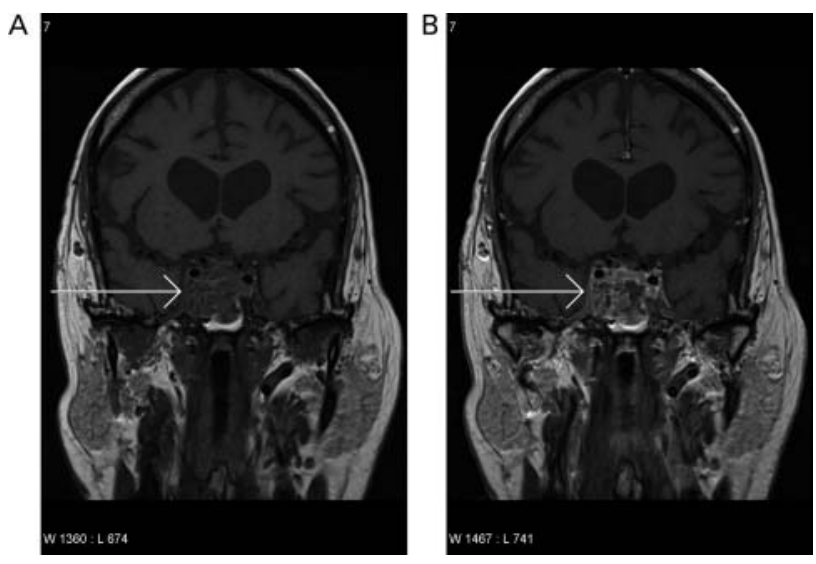

Figure 3

(A) Coronal T1 scan of the pituitary region showing a T1 isointense sellar, suprasellar and infrasellar lesions with invasion into the right cavernous sinus (arrow). (B) Coronal T1 post-gadolinium scan of the pituitary showing enhancement of the lesion (arrow). 
A

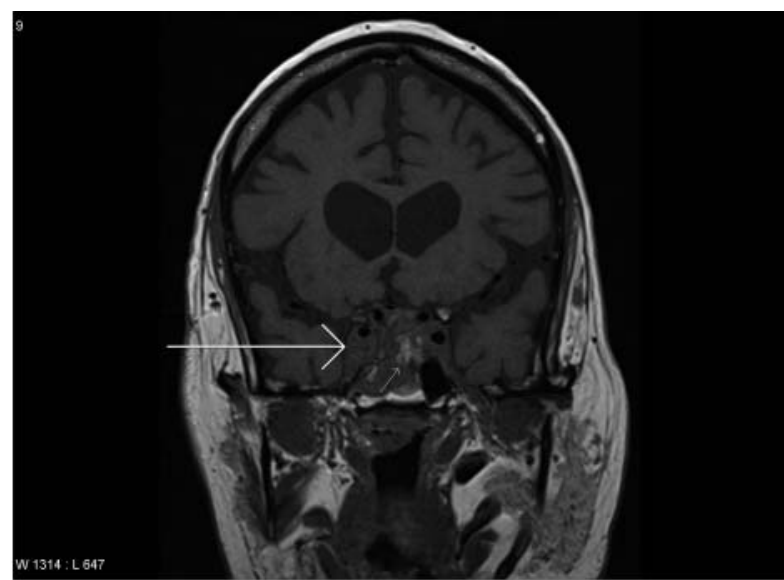

B

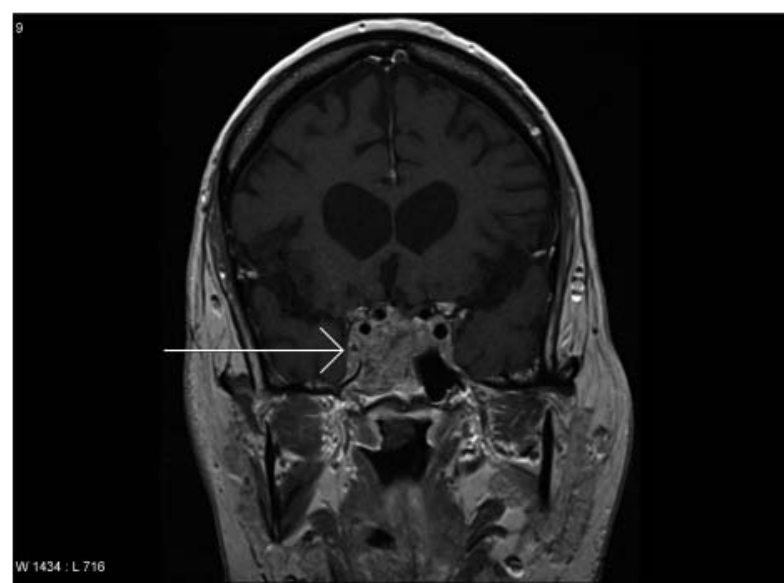

Figure 4

(a) Coronal T1 scan of the pituitary region showing rapid interval enlargement of the mass with new areas of increased T1 signal (small arrow) consistent with haemorrhage into tumour. There is progressive enlargement and invasion of the right cavernous sinus (large arrow). (B) Coronal T1 post-gadolinium scan showing a corresponding large enhancing mass invading the right cavernous sinus (large arrow) and now also invades the left cavernous sinus. Features are consistent with enlarging haemorrhagic tumour.

haemorrhage. Also MRI before second surgery demonstrated haemorrhage in the tumour mass. Moreover his clinical presentation was consistent with pituitary apoplexy due to intra-tumour haemorrhage.

\section{Sequential distinct pituitary neoplasms occurring in the same individual}

There are reports of 'collision sellar tumours' which is described as multiple tumours or pathological entities co-existing within the sella simultaneously. These are uncommon with Koutourousiou et al. (12) reporting eight cases out of 548 trans-sphenoidally resected pituitary adenomas. A case is described of Cushing's disease, which failed pituitary surgery and was managed with pituitary irradiation and bilateral adrenalectomy. He later presented with an aggressive null-cell pituitary carcinoma (13). However, our case highlights a novel situation of a non-functioning pituitary adenoma being followed by an aggressive non-adenomatous malignant spindle and round-cell tumour. Detailed histological examination of the pituitary neoplasms in our patient did not show any relationship between the two. There are several theories put forth to explain the existence of two pituitary pathologies in the same patient, but there is no proven mechanism (12). In particular, we could find no evidence that would link this tumour to any known genetic mutation predisposing to pituitary neoplasia such as the multiple endocrine neoplasia type 1 (MEN1) or aryl hydrocarbon receptor interacting protein $(A I P)$ genes.

Declaration of interest

The authors declare that there is no conflict of interest that could be perceived as prejudicing the impartiality of the research reported.

\section{Funding}

This research did not receive any specific grant from any funding agency in the public, commercial or not-for-profit sector.

\section{Patient consent}

Informed consent has been obtained from the patient's daughter for publication of the submitted article and accompanying images.

\section{Author contribution statement}

S Teasdale, Principal Author; F Hashem contributed to text; W J Inder, treating Endocrinologist during hospital admission, edited the manuscript; $S$ Olson treating, neurosurgeon; B Ong, Radiologist; permission was obtained from Endocrinologist Professor John Prins.

\section{References}

1 Rajasekaran S, Vanderpump M, Baldeweg S, Drake W, Reddy N, Lanyon M, Markey A, Plant G, Powell M, Sinha S et al 2011 UK guidelines for the management of pituitary apoplexy. Clinical Endocrinology 74 9-20. (doi:10.1111/j.1365-2265.2010.03913.x)

2 Pal A, Capatina C, Tenreiro AP, Guardiola PD, Byrne JV, Cudlip S, Karavitaki N \& Wass JA 2011 Pituitary apoplexy in non-functioning pituitary adenomas: long term follow up is important because of significant numbers of tumour recurrences. Clinical Endocrinology $\mathbf{7 5}$ 501-504. (doi:10.1111/j.1365-2265.2011.04068.x)

3 Robert CM, Jr, Feigenbaum JA \& Stern WE 1973 Ocular palsy occurring with pituitary tumors. Journal of Neurosurgery 38 17-19. (doi:10.3171/ jns.1973.38.1.0017)

4 Kim SH, Lee KC \& Kim SH 2007 Cranial nerve palsies accompanying pituitary tumour. Journal of Clinical Neuroscience 14 1158-1162. (doi:10.1016/j.jocn.2006.07.016) 
5 Lau KK, Joshi SM, Ellamushi H \& Afshar F 2007 Isolated bilateral oculomotor nerve palsy in pituitary apoplexy: case report and review. British Journal of Neurosurgery 21 399-402. (doi:10.1080/ 02688690701480710)

6 Walsh FB 1949 Bilateral total ophthalmoplegia with adenoma of the pituitary gland; report of two cases; an anatomic study. Archives of Ophthalmology 42 646-654. (doi:10.1001/archopht.1949.

00900050656014)

7 Keane JR 1984 Pituitary apoplexy presenting with epistaxis. Journal of Clinical Neuro-Ophthalmology 4 7-8.

8 Komurcu HF, Ayberk G, Ozveren MF \& Anlar O 2012 Pituitary adenoma apoplexy presenting with bilateral third nerve palsy and bilateral proptosis: a case report. Medical Principles and Practice 21 285-287. (doi:10.1159/000334783)
9 Liniker E \& Hyatt P 2009 Temporary bilateral oculomotor nerve palsy as the sole presenting sign of a pituitary mass. BMJ Case Reports 2193.

10 Mamelak N, Carmichael J, Bonert VH, Cooper O \& Melmed S 2013 Single-surgeon fully endoscopic endonasal transsphenoidal surgery: outcomes in three-hundred consecutive cases. Pituitary 16 393-401. (doi:10.1007/s11102-012-0437-1)

11 Chentli F 2013 Nasal bleeding due to huge prolactinomas. Acta Endocrinologica 9 473-478. (doi:10.4183/aeb.2013.473)

12 Koutourousiou M, Kontogeorgos G, Wesseling P, Grotenhuis P \& Seretis A 2010 Collision sellar lesions: experience with eight cases and review of the literature. Pituitary 13 8-17. (doi:10.1007/s11102-009-0190-2)

13 Goh KP, Lee HY \& Rajasoorya RC 2008 Triple jeopardy in the pituitary. Pituitary 11 331-336. (doi:10.1007/s11102-007-0075-1)

Received in final form 11 January 2015

Accepted 19 January 2015 